\title{
Risk Factors of Bloodstream Infections Caused by Carbapenem-Resistant Gram-Negative Pathogens in Pediatric Critical Care Settings
}

\author{
(1) Zümrüt Şahbudak Bal1, (1) Muhterem Duyu2, (1) Fulya Kamit3, (1) Pınar Yazici2, (1) Ayse Berna Anil4, \\ (1) Dilek Yilmaz Ciftdogan5, (1) Nisel Yilmaz Özkalay6, (1) Feriha Cilli7, (1) Bulent Karapinar2
}

'Ege University Faculty of Medicine, Department of Pediatrics, Division of Infectious Diseases, İzmir, Turkey 2Ege University Faculty of Medicine, Department of Pediatrics, Division of Intensive Care Unit, İzmir, Turkey

3University of Health Sciences, İzmir Tepecik Training and Research Hospital, Clinic of Pediatrics, Division of Intensive Care Unit, İzmir, Turkey ${ }^{4}$ izmir Katip Celebi University Faculty of Medicine, Department of Pediatrics, Division of Intensive Care Unit, izmir, Turkey 5 İzmir Katip Celebi University Faculty of Medicine, Department of Pediatrics, Division of Infectious Diseases, izmir, Turkey ${ }^{6}$ University of Health Sciences, Izmir Tepecik Training and Research Hospital, Clinic of Clinical Microbiology and Infectious Diseases, İzmir, Turkey ${ }^{7}$ Ege University Faculty of Medicine, Department of Clinical Microbiology and Infectious Diseases, İmir, Turkey

\begin{abstract}
Aim: Infections and sepsis are the leading causes of death in non-cardiac ICUs and account for 40 percent of all ICU expenditures. Data regarding bloodstream infections (BSIs) due to a carbapenem-resistant gram negative (CRGN) microorganis in pediatric ICUs still remain limited.

Materials and Methods: This study was conducted retrospectively in patients who were admitted to two pediatric critical care units between January 2011 and December 2017. Patients were assigned to two groups. Patients with bloodstream infection (BSI) caused by a carbapenem-resistant gramnegative microorganism and infections were assigned to BSI group other than BSI were assigned to non-BSI group.

Results: This study included 96 critically ill children with a mean age of 48.6 (1-204) months. Requirement of invasive procedures included tracheostomy, foley catheter and central venous catheter were not statistically different among groups, P values were 0.159, 0.291 and 0.803 , respectively. The majority of the patients admitted to PICU due to sepsis/septic shock in BSI group (n: 18,58\%) and in non-BSI group (n: 24, 37,9\%). Prior third/fourth generation cephalosporin exposure significantly more common in BSI group ( $51.6 \%$ vs.15.5\%, $\mathrm{P}<0.001$ ), carbapenem exposure was not significantly different among groups $(35.5 \%$ vs $56.9 \%, P=0.054)$. Neutropenia $\left(<500 / \mathrm{mm}^{3}\right)$ and thrombocytopenia $\left(150 \times 103 / \mathrm{mm}^{3}\right)$ were more significantly more common in BSI group ( $P=0.011$ and $P=0.010)$ and $C$-reactive protein $(C R P)$ level was significantly higher $(P=0.018)$. Crude and attributable mortality did not show significance between groups, $P$ values were 0.578 and 0.955 , respectively.

Conclusion: Carbapenem -resistant gram-negative infections are still major cause of morbiditity, mortality and healthcare associated infections. In this sudy, we evaluated the

patients with BSI due to a CRGN microorganism and compared with other infection types. The risk factors and outcomes were similar except prior cephalosporin exposure. As a conclusion, we have to enhance the infection control programs and prevent the patients from redundant antibiotic exposure.

Keywords: Carbapenem-resistant gram-negative microorganism, bloodstream infection, pediatric critical care unit
\end{abstract}

\section{Introduction}

Antibiotic resistance across gram-negative bacteria has progressively disseminated to countries worldwide, presenting a serious public health concern. Although intensive care units (ICUs) account for fewer than 10 percent of total beds in most hospitals, majority of all healthcare associated infections occurred in ICU Departments of hospitals (1, 2). Infections and sepsis are the leading causes of death in non-cardiac ICUs and account for 40 percent of all ICU 
expenditures (3). A recent review evaluated carbapenemresistant Enterobacteriaceae (CRE) infections reported that the majority of children (53\%) acquired carbapenem-resistant gram negative (CRCN) infections during ICU stay (4). Vincent IL et al. (3) concluded that infections have been common in patients in contemporary ICUs, and longer duration hospital stay correlated with risk of infection. Infections due to CRE in adult populations have been associated with poor clinical outcomes, including mortality rates as high as $40-65 \%$ while mortality can reach up to $90 \%$ in children $(4,5)$. Data regarding bloodstream infections (BSIs) due to a CRGN pathogen in pediatric ICUs still remain limited $(6,7)$. Therefore, we evaluated the clinical and laboratory features of BSIs caused by a CRGN pathogen and compared with other type of infections included urinary tract infection (UTI), ventilator-associated pneumonia (VAP) and meningitis.

\section{Material and Methods}

This study was conducted retrospectively in patients who were admitted to two pediatric critical care units between January 2011 and January 2017. Demographic characteristics, medical history, comorbidity, pathogens isolated and antimicrobial susceptibility of isolated pathogens, treatment administered, administration of other nephrotoxic agents, duration of PICU stay before isolation of resistant microorganisms, presence of medical devices, such as central catheters, urinary catheters, and endotracheal or tracheostomy tubes, ventriculo-peritoneal device were recorded retrospectively from medical records.

\section{Microbiologic Testing}

Presumptive identification of Gram-negative pathogens was identified by VITEK MS (bioMérieux, France). Vitek-MS that is using Matrix assisted laser desorption ionization time of flight mass spectrometry (MALDI-TOF MS) technology which is a new technology for species identification based on the protein composition of microbial cells. The isolate was tested for antibiotic sensitivity on Muller Hinton agar by Kirby Bauer disc diffusion technique using standard methods. The susceptibilities of amikacin, ceftriaxone, ceftazidime, piperacillin-tazobactam, cefoperazone-sulbactam, imipenem, meropenem, colistin and tygecycline were determined according to Clinical and Laboratory Standards Institutes (CLSI) guidelines. Definitions

We reviewed the medical records of the enrolled patients and collected the case information. A standard form was used to collect the epidemiologic data including age, sex, underlying diseases (pulmonary disease, malignancy, cardiovascular disease, hematologic/solid organ transplantation, metabolic disease, genetic syndrome, prematurity, renal disease, liver disease), medication or intervention (presence of tracheal cannula, central venous catheter, presence of a Foley catheter, mechanical ventilation, immunosuppressive therapy and steroid, receipt of antibiotics). Diagnosis of infection was based on clinical features and isolation of bacteria from normally sterile site. A patient with CRGN BSI was defined as BSI group and the patients with other infection types such as VAP, UTI and meningitis were included in non-BSI group. Standard definitions of nosocomial infections and ventilator associated pneumonia (VAP) were used according to the Center for Disease Control and Prevention (CDC) definitions (13) and diagnosis of sepsis was made according to International Pediatric Sepsis Consensus (14).

Crude mortality was defined if a patient died 1 month within infection and attributable mortality was defined if a patient died directly related to breakthrough infection.

\section{Statistical Analysis}

Statistical analyses were performed using SPSS for Windows (version 22.0; SPSS, Inc., Chicago, IL, USA]. Numerical data were expressed as medians (interquartile range). Mann-Whitney $U$ and Wilcoxon tests were used for inter-variable analysis. Categorical variables were evaluated with the Chi-square test or the two-tailed Fisher exact test and presented as percentages in acquiring CRGN infections. Comparisons were referred to as statistically significant if the P-values were $<0.05$.

\section{Ethics}

This study had the permission of the Ethical Board of Izmir Katip Celebi University [ethical decision number:58/ March 24,2016].

\section{Results}

This study included 96 critically ill children with a mean age of 48.6 (1-204) months between January 2011 and December 2017. The most common type of infection was VAP (59 cases, 66,2\%), followed by BSI (31 cases, 39.6\%), UTI (10 cases, $11.2 \%$ ), meningitis ( 3 cases, $3.3 \%$ ). Both bloodstream infection and VAP occurred in 7 (7.8\%) patients, BSI and UTI occurred $3(3.3 \%)$ patients and VAP and UTI occurred in 3 (3.3\%) patients. The most common underlying disease was chronic neurological disorder in BSI group (n:9, 29\%) and non-BSI groups ( $n: 18,31 \%)$ and followed by bone marrow/ solid organ transplantation ( $\mathrm{n}: 7,22.5 \%$ ) while chronic lung diseases ( $\mathrm{n}: 9,15.5 \%$ ) in non-BSI group (Table I). The majority of the patients admitted to PICU due to sepsis/septic shock in BSI group (n:18, 58\%) and in non-BSI group (n:24, 37,9\%). Requirement of invasive procedures included tracheostomy, foley catheter and central venous catheter were not statistically different among groups, P values were 0.159 , 0.291 and 0.803 , respectively. The mean age of the patients significantly younger in BSI group than non-BSI group (12 vs.32, $\mathrm{P}=0.022$ ). Total and prior hospital stay did not show significant difference, $\mathrm{P}$ levels were 0.384 and 0.803 , respectively. Prior third/fourth generation cephalosporin exposure significantly 
more common in BSI group (51.6\% vs.15.5\%, $P<0.001)$, carbapenem exposure was not significantly different among groups (35.5\% vs 56.9\%, P=0.054). Crude and attributable mortality did not show significance between groups, $P$ values were 0.578 and 0.955 ,respectively. Neutropenia $\left(<500 / \mathrm{mm}^{3}\right)$ and thrombocytopenia $\left(150 \times 103 / \mathrm{mm}^{3}\right)$ were more significantly more common in BSI group $(P=0.011$ and $\mathrm{P}=0.010$ ) and $\mathrm{C}$-reactive protein (CRP) level was significantly higher $(P=0.018)$. White blood cell, absolute neutrophil count, hemoglobin level did not show statistical significance, $P$ levels were $0.271,0.121$ and 0.822 , respectively.

\section{Discussion}

In this study, a retrospective case-control study was conducted to evaluate the risk factors for acquisition of BSI caused by CRGN microorganism and to compare with other infection types other than BSI in patients those admitted to PICUs of two teaching hospitals. We found BSIs occurred in younger patients and thrombocytopenia and neutropenia were significantly more common in BSI group. The crude and attributable mortality rate did not show statistical significance between the groups. Over recent years, CRGN

Table I. Risk factors and outcomes of Carbapenem-resistant gram negative bloodstream infections and comparision with non-bloodstream infections group

\begin{tabular}{|c|c|c|c|}
\hline & BSI (n:31) & Non BSI (n:58) & $P$ value \\
\hline Age, months, mean $\pm S D$ & $32.8( \pm 37.04)$ & $66.7( \pm 72.9)$ & 0.022 \\
\hline Hospital stay prior to BSI (median, \pm SD) (day) & $17(9.5-128.1)$ & $10(11.6-21.8)$ & 0.803 \\
\hline Total hospital stay(median, \pm SD) (day) & $27(2.89-168.65)$ & $34(34.2-56.9)$ & 0.384 \\
\hline Underlying diseases $(n, \%)$ & & - & 0,029 \\
\hline Previously healthy & $1(3.2)>$ & $12(20.7)$ & \\
\hline Chronic neurological/neuromuscular disorder & $9(29)$ & $18(31)$ & \\
\hline Hematologic/solid malignancy & $1(3.2)$ & $3(5.2)$ & \\
\hline Chronic liver diseases & $1(3.2)$ & $4(6.9)$ & \\
\hline Chronic lung disease & $2(6.5)$ & $9(15.5)$ & \\
\hline Congenital heart disease & $4(12.9)$ & $6(10.3)$ & \\
\hline Bone marrow/solid organ transplantation & $7(22.6)$ & $2(3.4)$ & \\
\hline Primary immune deficiency & $3(9.7)$ & $3(5.2)$ & 0,165 \\
\hline Others & $3(9.7)$ & $1(1.7)$ & \\
\hline Cause of PICU admission $(n, \%)$ & - & - & \\
\hline Sepsis/Septic shock & $18(58.1)$ & $24(41.4)$ & \\
\hline Respiratory failure & $10(32.3)$ & $22(37.9)$ & \\
\hline Trauma & $1(3.2)$ & $9(15.5)$ & \\
\hline Status epilepticus & 0 & $2(3.4)$ & \\
\hline $\begin{array}{l}\text { Cardiopulmonary arrest } \\
\text { Tracheostomy }(n, \%) \\
\text { Foley catheter }(n, \%) \\
\text { Central venous catheter }(n, \%)\end{array}$ & $\begin{array}{l}2(6.5) \\
10(32.3) \\
24(77.4) \\
29(93.5)\end{array}$ & $\begin{array}{l}1(1.7) \\
11(19) \\
50(86.2) \\
55(94.8)\end{array}$ & $\begin{array}{l}0.159 \\
0.291 \\
0.803\end{array}$ \\
\hline Thrombocytopenia $\left(<150 \times 103 / \mathrm{mm}^{3}\right)(\%)$ & $13(50)$ & $13(22.4)$ & 0.031 \\
\hline Neutropenia $\left(<500 / \mathrm{mm}^{3}\right)(\%)$ & $4(13.8)$ & $0(0)$ & 0.011 \\
\hline WBC $\left(\mathrm{mm}^{3}\right)$ & 13100 (7677-16018) & $12600(12058-16438)$ & 0.207 \\
\hline ANC $\left(\mathrm{mm}^{3}\right)$ & $7250(5040-12477)$ & $9140(8528-12564)$ & 0.105 \\
\hline $\operatorname{PLT}\left(x 10^{3} / \mathrm{mm}^{3}\right)$ & $212(140153-298944)$ & $315(285627-399193)$ & 0.010 \\
\hline $\mathrm{CRP}(\mathrm{mg} / \mathrm{dL})$ & $9.65(7.47-12.5482)$ & $7.3(5.43-82051)$ & 0.018 \\
\hline Attributable mortality $(n, \%)$ & $5(16.1)$ & $11(19)$ & 0.740 \\
\hline Crude mortality $(n, \%)$ & $5(16.1)$ & $18(\% 31)$ & 0.126 \\
\hline
\end{tabular}


infections have been accused as a significant cause of healthcare associated infections, significant mortality and morbidity. Previous adult studies suggested that elder patients are more vulnerable to CRE infections. On the other hand, a recent study from UK evaluating CRE infections, demonstrated majority of the patients $(6 / 9 ; 66.6 \%)$ were under 1 year of age (8). Similarly, we found that patients with BSI younger than the patients with other types of infection.

A recent study evaluated the risk factors and clinical outcomes of patients with CR Acinetobacter baumanni bacteremia and found that the independent risk factors were hematological malignancy, previous cefepim exposure and the use of total parenteral nutrition (9). Routsi et al. (10) suggested prior exposure to carbapenems were independent risk factors for acquisition of CR-GNB. Previous administration of carbapenems was the only factor related with the development of CR-GNB if the source of infection was other than VAP. Patients with bacteremia were more likely to have additional devices and longer hospital stay. In the present study, carbapenem exposure rate was similar among groups while prior cephalosporin 3-fold more common in BSI group when compared with non-BSI group $(<0.001)$.

A recent mataanalysis evaluating 9 studies reported that death rates were higher in patients had bacteremia caused by a CRGN pathogen when compared with a CSCN pathogen. However, they suggested no difference between groups; bacteremia and other infections (11). We compared the patients with BSI caused by a CRGN pathogen and other types of infection due to a CRGN pathogen and not found a significant difference. A case control study has been used to identify the risk factors and compare the outcomes (12). Previous studies demonstrated 3-6 times higher mortality among CRE-infected patients when compared with Carbapenem-sensitive Enterobacterieaceae infected patients (13-15). We did not find significant difference among groups.

Acquired risk factors for CRE infection in children include underlying chronic medical conditions, invasive medical devices, frequent or prolonged hospitalizations, prior antibiotic exposure, age, and travel from endemic regions (13). Among BSI and non-BSI groups, prior and total hospital stay duration did not show difference.

Thrombocytopenia is a common finding in patients with bacterial sepsis as a result of marrow suppression, consumption due to DIC and inflammatory response, and/or drug-induced. A recent study compared methicillin-resistant and methicillin-sensitive S.aureus infected patients and found no significant difference for thrombocytopenia (16). A recent multicenter observational study demonstrated that thrombocytopenia within the first 24 hours of septic shock onset as a prognostic marker of survival at day 28 in a large cohort of ICU patients (17). We found that patients in BSI group significantly more likely to develop thrombocytopenia as previous reports.
There are several advantages of this study. The main advantage is to be the largest series in pediatric critical care settings and to be the first study that evaluates the impact of having a BSI due to a CRGN pathogen and multicenter design. The main limitations of this study were the retrospective design and lack of molecular characterization of microorganisms which can affect the outcomes of these infections. We additionally defined CRGN as an organism demonstrating resistance to at least one carbapenem antibiotic and therefore could not assess the risk factors paticularly for carbapenemase-producing CRCN, which may be distinct from those for non-carbapenemase-producing CRGN.

CRGN infections are still major cause of morbidity, mortality and healthcare associated infections. In this study, we evaluated the patients with BSI due to a CRGN microorganism and compared with other infection types. The risk factors and outcomes were similar except prior cephalosporin exposure.

\section{Conclusions}

We have to enhance the infection control programs and prevent the patients from redundant antibiotic exposure. The current knowledge's are based on adult studies due to lack of prospective and case-control design studies. Therefore, further larger and prospective design studies are needed.

\section{References}

1. MacVane $\mathrm{SH}$. Antimicrobial Resistance in the Intensive Care Unit: A Focus on Gram-Negative Bacterial Infections. J Intensive Care Med. 2017;32(1):25-37.

2. Fridkin SK, Welbel SF, Weinstein RA. Magnitude and prevention of nosocomial infections in the intensive care unit. Infect Dis Clin North Am. 1997;11(2):479-96.

3. Vincent IL, Rello I, Marshall J, Silva E, Anzueto A, et al; EPIC II Group of Investigators. International study of the prevalence and outcomes of infection in intensive care units. JAMA. 2009 Dec 2;302(21):2323-9.

4. Logan LK. Carbapenem-resistant enterobacteriaceae: an emerging problem in children. Clin Infect Dis. 2012;55(6):852-9.

5. Chiotos K, Han JH, Tamma PD. Carbapenem-Resistant Enterobacteriaceae Infections in Children. Curr Infect Dis Rep. $2016 ; 18(1): 2$.

6. Siddiqui NU, Qamar FN, Jurair H, Haque A. Multi-drug resistant gram negative infections and use of intravenous polymyxin $B$ in critically ill children of developing country: retrospective cohort study. BMC Infect Dis. 201428;14:626.

7. Chiotos K, Tamma PD, Flett KB, Naumann M, Karandikar MV, Bilker WB, Zaoutis T, Han JH. Multicenter Study of the Risk Factors for Colonization or Infection with CarbapenemResistant Enterobacteriaceae in Children. Antimicrob Agents Chemother.2017;61(12)

8. Drew RJ, Turton JF, Hill RL, Livermore DM, Woodford N, Paulus S, Cunliffe NA. Emergence of carbapenem-resistant Enterobacteriaceae in a UK paediatric hospital. ) Hosp Infect. 2013;84(4):300-4. 
9. Huang ST, Chiang MC, Kuo SC, Lee YT, Chiang TH, et al. Risk factors and clinical outcomes of patients with carbapenemresistant Acinetobacter baumannii bacteremia. I Microbiol Immunol Infect. 2012 ; 45(5):356-62.

10. Routsi C, Pratikaki M, Platsouka E, Sotiropoulou C, Papas V, et al. Risk factors for carbapenem-resistant Gram-negative bacteremia in intensive care unit patients. Intensive Care Med.2013;39(7):1253-61.

11. Falagas ME, Tansarli CS, Karageorgopoulos DE, Vardakas KZ. Deaths attributable to carbapenem-resistant Enterobacteriaceae infections. Emerg Infect Dis. 2014;20(7):11705.

12. Ozsurekci Y, Aykac K, Cengiz AB, Basaranoglu ST, Sancak B, et al. Bloodstream infections in children caused by carbapenemresistant versus carbapenem-susceptible gram-negative microorganisms: Risk factors and outcome. Diagn Microbiol Infect Dis. 2017;87(4):359-364.

13. Patel G, Huprikar S, Factor SH, Jenkins SG, Calfee DP. Outcomes of carbapenem-resistant Klebsiella pneumoniae infection and the impact of antimicrobial and adjunctive therapies. Infect Control Hosp Epidemiol. 2008;29(12):1099-106.

14. Bleumin D, Cohen MJ, Moranne O, Esnault VL, Benenson S, Paltiel O, Tzukert K, Mor-Yosef Levi I, Ben-Dov IZ, Levi R, Bloch A, Haviv YS. Carbapenem-resistant Klebsiella pneumoniae is associated with poor outcome in hemodialysis patients. I Infect. 2012 Oct;65(4):318-25.

15. Ben-David D, Kordevani R, Keller N, Tal I, Marzel A, et al. Outcome of carbapenem resistant Klebsiella pneumoniae bloodstream infections. Clin Microbiol Infect. 2012 ;18(1):54-60.

16. Wang JT, Hsu LY, Lauderdale TL, Fan WC, Wang FD. Comparison of Outcomes among Adult Patients with Nosocomial Bacteremia Caused by Methicillin-Susceptible and Methicillin-Resistant Staphylococcus aureus: A Retrospective Cohort Study. PLoS One. 2015;10(12):e0144710.

17. Thiery-Antier $N$, Binquet $C$ Vinault $S$, Meziani F, BoisraméHelms I, Quenot JP; EPIdemiology of Septic Shock Group. Is Thrombocytopenia an Early Prognostic Marker in Septic Shock? Crit Care Med. 2016;44(4):764-72. 